\title{
Early diagnosis for cutaneous malignant melanoma based on the intellectualized classification and recognition for images of melanocytic tumour by dermoscopy
}

\author{
Ru-Song Meng ${ }^{1}$, Xiao Meng ${ }^{2}$, Feng-Ying $\mathrm{Xie}^{3}$, Zhi-Guo Jiang ${ }^{3}$ \\ 1. Department of Dermatology, General Hospital of the Air Force, PLA. 2. Guang' anmen Hospital, China Academy of \\ Chinese Medical Sciences, Beijing. 3. Image Processing Center, BeiHang University. \\ Correspondence: Ru-Song Meng. Address: No.30 Fu Cheng Road, Haidian District, Beijing, China. Telephone: \\ 86-108-851-9699. E-mail: mrs5822@sohu.com.
}

Received: March 31, 2012

DOI : $10.5430 /$ jbgc.v2n2p37
Accepted: June 6, 2012

Published: December 1, 2012

URL: http://dx.doi.org/10.5430/jbgc.v2n2p37

\section{Abstract}

Background: In recent years, the morbidity of Melanocytic Tumor, especially Cutaneous Malignant Melanoma, has been increasing year by year. Cutaneous Malignant Melanoma has been the most fatal skin disease owing to its high malignant level and proneness to metastasis. It is of great importance to establish the intellectualized classification and recognition for Melanocytic Tumor in the yellow race, in order to realize the early diagnosis and reduce the mortality for Cutaneous Malignant Melanoma.

Methods: We adopted the polarized-light dermoscopy image technology; acquired the images of Melanocytic Tumor from the yellow race in a non-invasive way; proposed the algorithms of adaptive clustering segmentation and feature extraction; quantificationally analyzed the six features of Melanocytic Tumor images, including its asymmetry, eccentricity, border depressed rate, uniformity of radiation in transition area, color diversity and texture correlation; realized the classification and intellectualized recognition for benign or malignant Melanocytic Tumor, with the combined neural network classifier; verified the results by histopathology and statistical analysis.

Results: Among the 642 images, the benign ones accounted for $82.4 \%$, the malignant ones $17.6 \%$. The sensitivity and the specificity of uniformity of radiation in transition area, texture correlation, border depressed rate and color diversity were from $86.73 \%$ to $95.58 \%$, and from $97.3 \%$ to $100 \%$ respectively, which showed relatively high performance. The sensitivity and the specificity of asymmetry and eccentricity were from $41.59 \%$ to $47.78 \%$, and from $69.91 \%$ to $76.99 \%$ respectively, which showed relatively low performance. The average classification accuracy for benign and malignant Melanocytic Tumor reached $93.65 \%$ by the multiple independent neural network classifiers, the difference of which was significant by chi-square test $\left(\chi^{2}=4.51, P<0.05\right)$.

Conclusions: The polarized-light dermoscopy analysis technology is able to realize the feature classification and automatic recognition for benign or malignant Melanocytic Tumor in a non-invasive way. It will lay foundations for solving the key problem of early diagnosis and intellectualized recognition for Cutaneous Malignant Melanoma in the yellow race. 


\section{Key words}

Polarized-light dermoscopy image, Image segmentation, Feature recognition, Combined neural network classification, Melanocytic tumor

\section{Introduction}

Cutaneous Melanocytic Tumor (MT) includes benign tumor, namely Melanocytic Nevus (MN), and malignant tumor, namely Cutaneous Malignant Melanoma (CMM). According to the statistics from the National Cancer Institute of The United States, the incidence of skin tumors, especially CMM, has been increasing all over the world.

CMM has been the most fatal skin disease owing to its high malignant level and proneness to metastasis ${ }^{[1]}$. The $\mathrm{ABCD}$ has still been an often-used clinical method for the diagnosis of CMM, where A corresponds to asymmetry, B is border (irregular), $\mathrm{C}$ refers to color, and $\mathrm{D}$ corresponds to diameter (greater than $6 \mathrm{~mm}$ ). Since it is judged by human eyes, the diagnostic accuracy is only $67.26 \%$. Although the pathological diagnosis is regarded as gold standard, it easily causes blood or lymphatic metastasis in addition to severe trauma, which will undoubtedly increase the mortality of $\mathrm{CMM}^{[2,3]}$. The incidence of CMM in China is lower than that in other countries, but it has been increasing by 3\% to $8 \%$ year after year ${ }^{[2]}$, directly threatening the human beings' health and life. Ashfaq A et al. ${ }^{[4]}$ introduced the DB-Dermo Mips ${ }^{\circledR}$, a dermoscopy image computer-aided diagnosis system for CMM; it mainly focused on the image segmentation and automatic recognition for CMM in the white race; the sensitivity was $93 \%$ to $99 \%$; the specificity was $81 \%$ to $93 \%$. Because of the various complexions from different ethnics, the reflection factors of image information are diverse ${ }^{[5,6]}$. It will lead to significant diversity in the algorithms of image segmentation and feature extraction for CMM, which will directly influenced the precision of multiple quantitative parameters, the establishment of diagnostic standard and automatic recognition for $\mathrm{CMM}^{[7]}$.

In our study, we will adopt the technology of polarized-light dermoscopy image analysis; acquire the images of MT from the yellow race in a non-invasive way; propose the algorithms of adaptive clustering segmentation, feature extraction and quantitative analysis; realize the classification for benign or malignant MT; verify the classification results by histopathology and statistical analysis. This study may be reported for the first time in the international, which will lay foundations for solving the key problem of intellectualized recognition for CMM in the yellow race ${ }^{[7,8]}$.

\section{General data}

Cases, in accordance with the diagnostic standard of melanocytic lesion, were randomly collected in our hospital from June 2006 to June 2011, including congenital and acquired melanocytic lesions.

Among the 642 qualified cases, 529 cases were MN, including 158 males and 371 females; the mean age was (52.4 \pm 44.9$)$ years ranging from one to 87 years. While 113 cases were CMM, including 34 males and 79 females; the mean age was (58.7 \pm 50.4 ) years ranging from 21 to 87 years. The minimum and maximum diameters of lesions were $0.3 \mathrm{~cm}$ and $7.34 \mathrm{~cm}$ respectively.

\section{Instruments}

The Dermoscopy- II Image Diagnosis Workstation, jointly developed by Air Force General Hospital and Beijing Aerospace University, was adopted in our study and was abbreviated as Dermoscopy- II below. The polarized-light dermoscopy image technology ${ }^{[9,10]}$ was used to obtain the images of MT in a non-invasive way. Then the processes of segmentation, feature extraction, classification and recognition for the images were completed by the computer. 


\section{Methods}

The patients, who were suspected with MT, were diagnosed by experienced clinicians at first. For the qualified cases, the information, such as history of the disease, gender, age and locations of the lesions, were recorded in detail. Then the technicians examined the lesions in target areas by Dermoscopy- II ; performed image acquisition, storage and management; analyzed these images; divided them into suspected benign group and suspected malignant group according to the results. Meanwhile, biopsy was taken for the lesions in the same target area to make pathological diagnosis, and then the cases were classified into benign group and malignant group as well. The results between different groups were dealt with by experimental evaluation and statistical analysis, in order to ascertain the recognition rate, sensitivity and specificity for CMM by Dermoscopy- II .

\subsection{The intellectualized image segmentation, feature extraction, classi- fication and recognition for CMM by Dermoscopy- II}

(1) The algorithm of image segmentation for CMM in the yellow race ${ }^{[7]}$

The adaptive clustering algorithm, based on the combination of self-generating neural network (SGNN) and improved genetic algorithms (IGAs), was adopted in our study. The algorithm involved three major steps: first, a group of optimal seed samples were selected through IGAs; second, these seed samples were taken as initial trees to generate the self-generating neural forest (SGNF) by training the rest samples based on SGNN; finally, every tree in the SGNF may denote a cluster in melanocytic tumor image to complete the clustering segmentation.

(2) The method of feature extraction

The feature extraction and quantitative analysis were performed according to the six features for images of CMM, including its asymmetry (AY), eccentricity (EY), border depressed rate (BDR), uniformity of radiation in transition area (URTA), color diversity (CD) and texture correlation (TC).

\section{$A Y$}

In order to evaluate the AY of the lesion, first, the major axis orientation of the object $(\theta)$ is calculated by the formula below:

$$
\theta=\frac{1}{2} \tan ^{-1}\left(\frac{2 \mu_{11}}{\mu_{20}-\mu_{02}}\right)
$$

where $\mu_{p q}$ denotes the $(p+q)$ th order central moments of the object.

Second, the object is rotated $\theta$ degrees clockwise to align the principal (major and minor) axes with the image ( $\mathrm{x}$ and $\mathrm{y}$ ) axes. The object is then hypothetically folded about the $\mathrm{x}$-axis and the area difference $\left(A_{x}\right)$ between the overlapping folds is taken as the amount of asymmetry about the $\mathrm{x}$-axis. The same procedure is performed for the $\mathrm{y}$-axis. Two asymmetry measures are calculated from $A_{x}$ and $A_{y}$ as follows:

$$
\text { Asymmetry_Rate1 }=\frac{\min \left(A_{x}, A_{y}\right)}{A} \times 100 \%
$$




$$
\text { Asymmetry_Rate } 2=\frac{A_{x}+A_{y}}{A} \times 100 \%
$$

where $A$ is the area of the lesion. Figure 1 shows the process of evaluating the AY of the object.

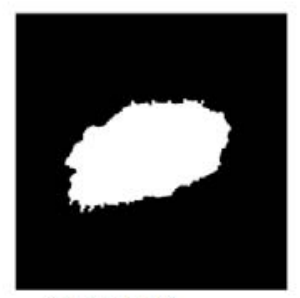

(a) the object

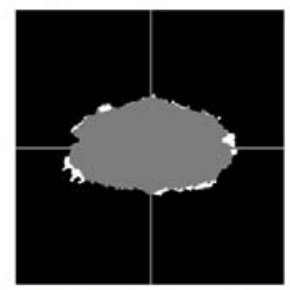

(c) hypothetically folded about the $x$-axis

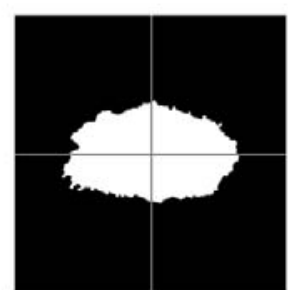

(b) rotated $\theta$ degrees

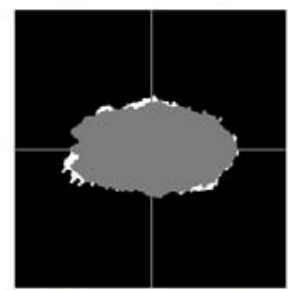

(d) hypothetically folded about the y-axis

Figure 1. Evaluating asymmetry of the object

$E Y$

It can be defined as the distance between the centre of an eccentric and the axis in the target area.

For the binary discrete image $f(i j)$, if the object is ' 1 ', background is ' 0 ', then the central moments of the image is:

$$
\mu_{p q}=\sum_{i} \sum_{j}\left(i-r_{0}\right)^{p}\left(j-c_{0}\right)^{q}
$$

where $(i, j)$ denotes the value of the rows and the columns, and $\left(r_{0}, c_{0}\right)$ denotes the object centroid in the target area. This function only reflects the shape of the object while ignoring its gray level detail. The EY of the object is defined as:

$$
\varepsilon=\frac{\left(\mu_{02}-\mu_{20}\right)^{2}+4 \mu_{11}}{\left(\mu_{02}+\mu_{20}\right)^{2}}
$$

$B D R$

For the BDR of one lesion $R_{\text {cancave }}$, the formula is defined as

$$
R_{\text {cancave }}=\frac{1}{n} \sum_{i=1}^{n} \frac{R A_{i}}{l_{i}}
$$


where $n$ denotes the number of depressed area of one object; $l_{i}$ is the span of the $i$ th depressed area; RA means the area of depressed border. Figure 2(b) shows more intuitive explanation. $R_{\text {cancave }}$ represents the average depth of all depressed area of one object.
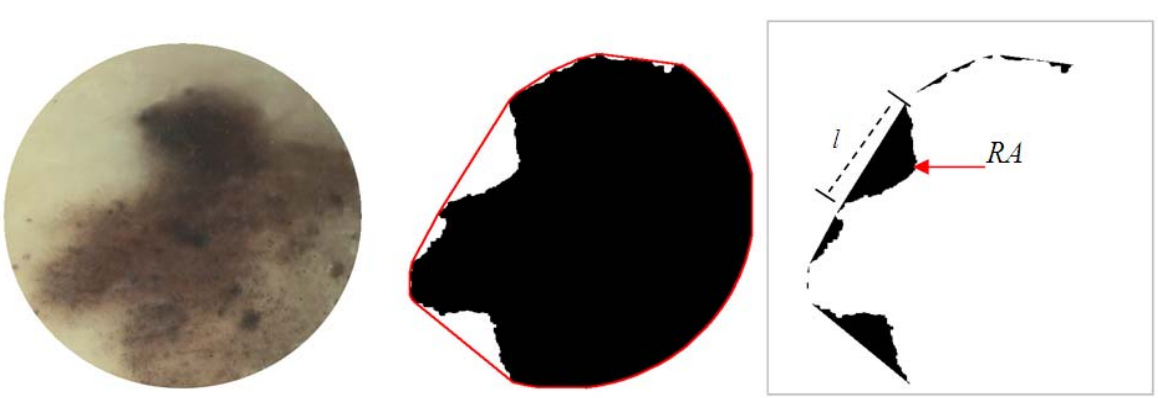

(a) Primitive image (b) The extracted target and concave-convex part (c) The algorithm of depressed rate

Figure 2. The diagram of BDR for CMM

URTA

$\Gamma_{\text {outer }}$ and $\Gamma_{\text {inner }}$ indicate pixel sets of outer and inner border respectively; $D\left(p_{i}, p_{j}\right)$ denotes the distance between pixel $p_{i}$ to $p_{j}$. Thus, the minimum distance from outer border pixel $p_{i}$ to inner border pixel $p_{j}$ is defined as:

$$
d_{i}=\min _{j}\left(D\left(p_{i}, p_{j}\right)\right), p_{i} \in \Gamma_{\text {outer }}, p_{j} \in \Gamma_{\text {inner }}
$$

The mean and variance of the distance from outer border pixel to inner border is defined as:

$$
\begin{gathered}
m=\frac{1}{n} \sum_{i=1}^{n} d_{i} \\
\delta=\sqrt{\frac{1}{n} \sum_{i=1}^{n}\left(d_{i}-m\right)^{2}}
\end{gathered}
$$

where $\mathrm{n}$ denotes the number of pixel of outer border. It indicates the dispersion of distance from outer border to inner border. The process of evaluating the URTA of the object is shown in Figure 6(c).

$C D$

$\mathrm{CD}$ is an important indicator to evaluate whether the lesion is malignant or benign. For 3-Dimention histogram computation of lesion, the RGB color space is coarsely quantized into $16 \times 16 \times 16$ bins. Defining $p(i, j, k)$ as $\mathrm{R} / \mathrm{G} / \mathrm{B}$ components statistical probability, the ColorNum distribution in the 3-Dimention histogram is defined as:

$$
\text { ColorNum }=\sum_{i=1}^{16} \sum_{j=1}^{16} \sum_{k=1}^{16} h(i, j, k)
$$




$$
\text { where } h(i, j, k)= \begin{cases}1, & \text { 当 } p(i, j, k) \neq 0 \\ 0, & \text { 当 } p(i, j, k)=0\end{cases}
$$

TC

Grey Level Co-occurrence Matrix (GLCM) $\hat{P}(i, j \mid d, \theta)$ reflects the general information of image grey level, involving orientation, neighborhood and magnitude of variance. The correlation of Co-occurrence Matrix indicates the orientation (row or column) similarity of elements of GLCM. The formula is defined as:

$$
C(d, \theta)=\frac{\sum_{i} \sum_{j} i j \hat{P}(i, j \mid d, \theta)-\mu_{1} \mu_{2}}{\sigma_{1}^{2} \sigma_{2}^{2}}
$$

where

$$
\begin{gathered}
\mu_{1}=\sum_{i} i \sum_{j} \hat{P}(i, j \mid d, \theta), \quad \mu_{2}=\sum_{j} j \sum_{i} \hat{P}(i, j \mid d, \theta) \\
\sigma_{1}^{2}=\sum_{i}\left(i-\mu_{1}\right)^{2} \sum_{j} \hat{P}(i, j \mid d, \theta), \quad \sigma_{2}^{2}=\sum_{j}\left(j-\mu_{2}\right)^{2} \sum_{i} \hat{P}(i, j \mid d, \theta)
\end{gathered}
$$

(3) The classification of MT based on combined neural network ${ }^{[7]}$

In accordance with the characteristics of MT images, two kinds of neural networks with different operating mechanisms, BP neural networks and fuzzy neural networks, were employed as elementary tools to design and train individual classifiers with Adaboost method. The combined classifier of integrating with multiple individual classifiers of different structure neural networks was implemented by optimizing and learning the combination weights of individual classifiers. The process is shown in Figure 3.

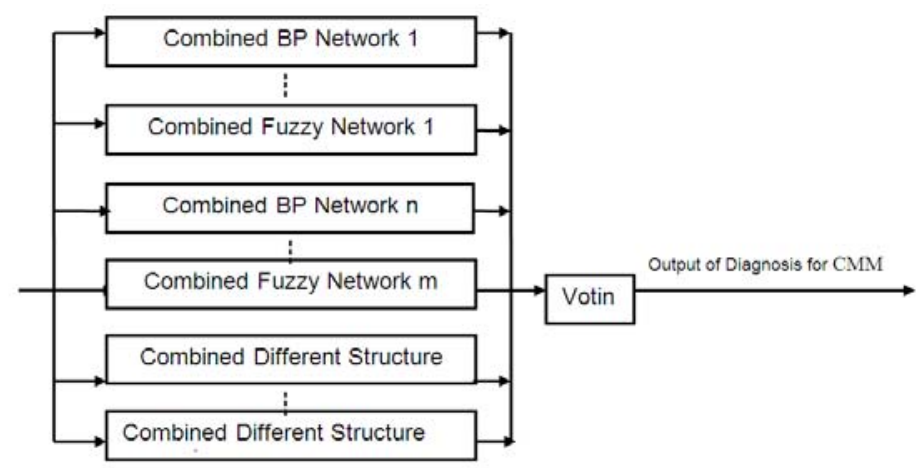

Figure 3. The integration of multiple combined neural

\subsection{Pathological observation}

(1) $\mathrm{MN}$

It revealed that the nevus cells were usually arranged in nests. The nevus cells, containing melanin inside, were classified into clear nevus cell, epithelial nevus cell, lymphoid nevus cell and spindle nevus cell. The pathological image of MN was shown in Figure 4. 


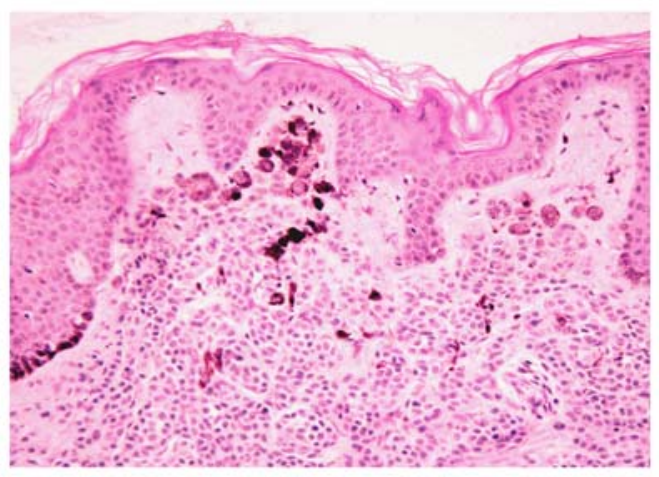

Figure 4. Pathological observation for MN by H.E X20

\section{(2) $\mathrm{CMM}$}

The tumour cells revealed various patterns, such as polygon, small circles, spindle shape, bubble, branching shape and peculiarly shape, with or without pigment. The cells contained nucleus with large, irregular nucleolus and mitotic phase. It could be divided into melanoma in situ and invasive melanoma. The pathological image of CMM was shown in Figure 5.

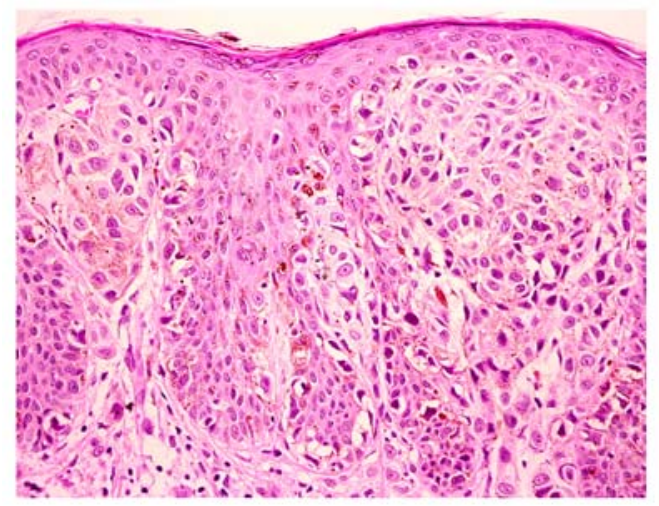

Figure 5. Pathological observation for CMM by H.E X20

\subsection{Statistical analysis}

We adopted Dermoscopy- II to test the characteristic conditions of URTA, TC, BDR, CD, AY and EY in MT, focusing on their occurrence and incidence in CMM. The data were analyzed by SPSS13.0 software and chi-square test was adopted for enumeration data. The difference was regarded as significant when $P<0.05$ at the significant level 0.05 .

\section{Results}

(1) After the process of image segmentation, feature extraction, combined neural network classification and data training for MT images by Dermoscopy- II, satisfactory results were acquired. Among the 642 cases of MT, 113 cases were CMM, accounted for $17.60 \%$; it was mainly used to test and describe the image features for CMM. Figure 6 was one of the examples of CMM, which was diagnosed as malignant lesion after the process by Dermoscopy- II . The cases of MN were 529, accounted for $82.40 \%$, and Figure 7 was the example which was diagnosed as benign lesion after the same process mentioned above. 


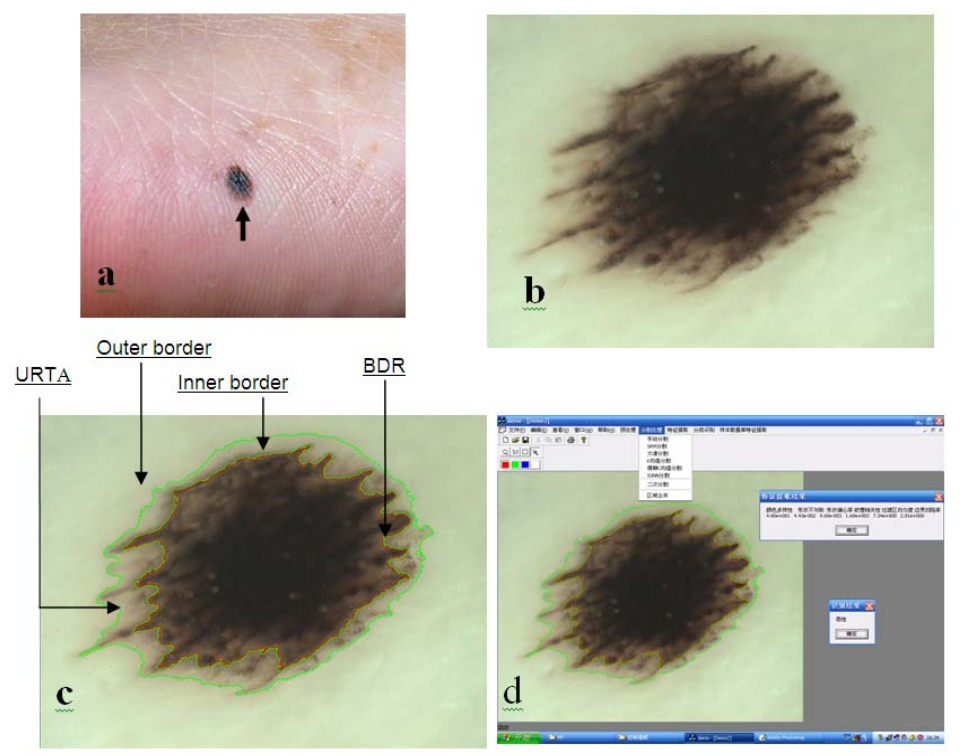

Figure 6. The lesion in voix pedis, diagnosed as CMM by Dermoscopy- II . (a) was a rough picture of CMM; (b) was the same image by dermoscopy; (c) revealed the state based on (b) after the segmentation by Dermoscopy- II ; (d) was diagnosed as CMM based on (b) by combined neural network classification.

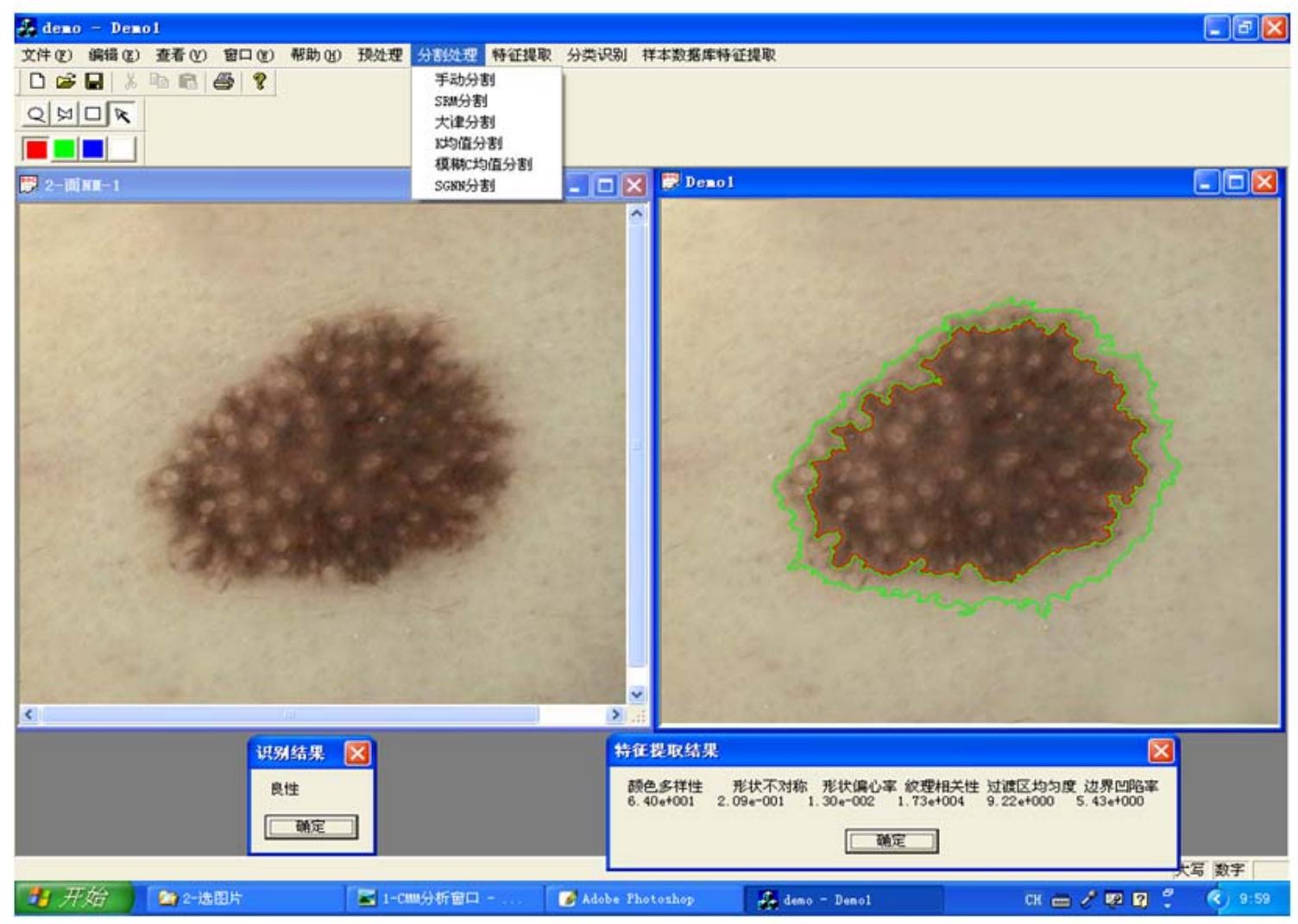

Figure 7. The lesion in face, which was diagnosed as benign tumor after the process of image segmentation, feature extraction and combined neural network classification by Dermoscopy- II . 
(2) The analyses on the sensitivity and the specificity of feature recognition for CMM images by Dermoscopy- II

The cases were analyzed in light of image features by Dermoscopy- II, and were compared with the diagnosis made by histopathology. Then the results were calculated by $2 \times 2$ table analysis, showing the sensitivity and the specificity of feature recognition by Dermoscopy- II. According to the results showed in Tab.1, the sensitivity and the specificity of URTA, TC, BDR and CD for CMM were from $86.73 \%$ to $95.58 \%$ and from $97.35 \%$ to $100 \%$ respectively, showing relatively high level. While the sensitivity and the specificity of AY and EY were from $41.59 \%$ to $47.78 \%$ and from $69.91 \%$ to $76.99 \%$ respectively, showing relatively low level.

Table 1. The analyses on the sensitivity and the specificity of feature recognition for 113 cases of CMM

\begin{tabular}{|c|c|c|c|c|c|c|c|}
\hline \multirow{2}{*}{$\begin{array}{l}\text { Image } \\
\text { feature by } \\
\text { dermoscopy }\end{array}$} & \multirow{2}{*}{$\begin{array}{l}\text { Classification } \\
\text { and recognition } \\
\text { by Dermoscopy- II }\end{array}$} & \multicolumn{2}{|c|}{ suspected benign } & \multicolumn{2}{|c|}{ suspected malignant } & \multirow{2}{*}{ sensitivity/\% } & \multirow{2}{*}{ specificity $/ \%$} \\
\hline & & benign & malignant & benign & malignant & & \\
\hline AY & & 79 & 66 & 34 & 47 & 41.59 & 69.91 \\
\hline EY & & 87 & 59 & 26 & 54 & 47.78 & 76.99 \\
\hline BDR & & 112 & 7 & 1 & 106 & 93.81 & 99.12 \\
\hline $\begin{array}{l}\text { URTA } \\
\text { transitional } \\
\text { region }\end{array}$ & & 110 & 15 & 3 & 98 & 86.73 & 97.35 \\
\hline $\mathrm{CD}$ & & 113 & 5 & 0 & 108 & 95.58 & 100 \\
\hline $\mathrm{TC}$ & & 111 & 9 & 2 & 104 & 92.04 & 98.23 \\
\hline
\end{tabular}

(3) The classification accuracy of MN and CMM, according to the image features of MT by Dermoscopy- II, with combined neural network classification

The 642 cases of MT were classified into MN group and CMM group by naked eyes, which were then verified by histopathology. Among the 113 cases of CMM, 76 cases were accordant in classification and 37 cases were not; its coincidence rate was $67.26 \%(76 / 113)$. While after the classification by the combined neural network of Dermoscopy- II , 106 cases were accordant in classification and 7 cases were not; the coincidence rate was $93.65 \%(106 / 113)$. According to the chi-square test, the coincidence rate of classification between the naked-eyes group and Dermoscopy- II group was significantly different $\left(\chi^{2}=4.51, P<0.05\right)$. The classification accuracy by Dermoscopy- II was $26.39 \%$ higher than that by naked eyes; its inconformity rate of classification was $6.19 \%(7 / 113)$, which might be caused by complicated or atypical features of CMM images by dermoscopy.

\section{Discussions}

At present, the most effective treatment for $\mathrm{CMM}$ is early recognition, early non-invasive diagnosis and timely resection, which is of decisive significance to guarantee the patients' health and to reduce mortality ${ }^{[2,3]}$. Consequently, the early diagnosis for CMM in a non-invasive way has been a globally popular topic.

Thomas P. Habif ${ }^{[11]}$ introduced that the ABCD rule for CMM by dermoscopy was first proposed by Stolz et al, namely the semi-quantitative method of asymmetry (A), border fuzziness (B), color variation (C) and different structural components(D); it played an important role in improving the diagnostic accuracy for CMM, but it was influenced by 
subjective factors. Ashfaq A et al ${ }^{[4]}$ introduced the DB-Dermo Mips ${ }^{\circledR}$, a dermoscopy image computer-aided diagnosis system for CMM, which has played a positive role in promoting the diagnosis for CMM. Since the automatic and precise segmentation for images is the critical technology for the realization of automatic recognition for $\mathrm{CMM}^{[7]}$, we adopted the polarized-light dermoscopy image technology in our study; acquired the images of CMM from the yellow race in a noninvasive way; proposed the algorithm of adaptive clustering segmentation based on SGNN; solved the problem about the sensitive training sequence of samples from CMM; confirmed the classification number adaptively without any manual intervention during the process; established the initial population scale for genetic algorithm at the same time, according to the size of the solution space; made dynamic regulation for genetic control parameters, including population scale, crossing-over rate and aberration rate, according to the individual changes during the evolutionary process, effectively improving the operating speed of algorithm and with good stability.

This study puts forward the six features of image recognition and quantitative analysis of MT in the yellow race, which are conclued as follows. (1)URTA: it represents the irregularity of CMM and non-uniformity of radiation, so the figure is relatively high. Comparatively, the width of strip in transitional region for $\mathrm{MN}$ is near and with uniform radiation, so the range and the variance are relatively small. (2)TC: the figure of $\mathrm{MN}$ is relatively high because of the regularity and uniformity of its texture; the figure of CMM is relatively low due to the large change of its texture. (3)BDR: it represents the average depth of all the depressed areas in one target of MT. Compared with MN, the depth of depressed area of CMM is larger, so it shows higher figure. (4) CD: because of the various colors in CMM, the figure is relatively high, while the figure of $\mathrm{MN}$ is low. (5) AY: the figure is relatively high because of the asymmetry of CMM lesion. (6)EY: the figure is higher due to the irregular shape of CMM, and the figure is relatively low because of the regular shape of MN. In this study, the images of CMM show higher performance in the aspects of URTA, TC, BDR and CD, which are the common characteristics for most CMM images; the sensitivity and the specificity reach from $86.73 \%$ to $95.58 \%$ and from $97.3 \%$ to $100 \%$ respectively; it plays an important value in the feature recognition for CMM. However, the sensitivity and the specificity of AY and EY are relatively low, which may be caused by the comprehensive statistical analysis for all the parameters in MT images. Because of the variety and complexity of the shapes and colors for CMM images, the manifestations in different anatomic locations are various. The morphological differences for MT images in palm plantar sites, where permanent friction or other physical stimulation is existed ${ }^{[4]}$, or in recurrent lesions, which are caused by improper physical therapy (laser or microwave treatment), are greater. The sensitivity and the specificity of AY and EY for these lesions are relatively low, showing little clinical significance for the classification and the recognition of MT. Except for the factors mentioned above, the sensitivity and the specificity of AY and EY for the other MT lesions are relatively high. In our study, the sample size of lesions in the palm plantar site or the recurrent MT lesions is very small, so independent statistical analysis can not be adopted. In our future work, the sample size will be enlarged; researches will be taken according to the lesions in different anatomic locations and the features of recurrent MT, in order to further improve the classification rate and the recognition rate for CMM.

For the sensitivity and the specificity of image features for CMM, we adopt combined neural network for the classification and the recognition of MT; the BP neural networks and fuzzy neural networks, which were employed as elementary tools to design and train individual classifiers with Adaboost method. The combined classifier of integrating with multiple individual classifiers of different structure neural networks is implemented by optimizing and learning the combination weights of individual classifiers ${ }^{[7]}$. In our study, the coincidence rate of classification reach $93.65 \%, 26.39 \%$ higher than that by naked eyes. The results has reached or exceeded the international research level in the white race. It has acquired satisfactory effect through experiment and clinical application.

Owing to the great quantity of the yellow race, the vastitude in territory and the complicated types of image features of $\mathrm{CMM}$, it is essential to increase the number of cases; to expand the source of database; to strengthen learning and optimized training on the multiple features of CMM by Dermoscopy- II, based on the methods mentioned above, in order to develop the classification rate and recognition rate of $\mathrm{CMM}$ and lay solid foundations for the intelligent recognition and early diagnosis for CMM. 


\section{Acknowledgement}

This work was supported by the National Natural Science Foundation of China (grant No.61027004, 61071138, 61271436).

\section{References}

[1] Wen-Jun Zhang, Hua Zhang.The development of mechanisms for Cutaneous Malignant Melanoma. Chin J Plast Surg. 2007; 23: 127-129.

[2] Fu-Ren Liu. Practical of Dermatology.Third edition. People hygiene press. 2005; 1034-1041.

[3] R.B. Odam,W.D.James,T.G.Berger.Andrews' diseases of the skin clinical dermatology.Ninth edition. Science and Technology Press. 2008; 737-742.

[4] Ashfaq A.Marghoob, Ralph P. Braun, Alfred W. Kopf. Atlas of dermoscopy. Composition by parthenon publishing. 2005; 19: 321.

[5] Markolf H Niemz. Laser tissue interaction principle and Application. Xi'an Jiao Tong University press. First edition. 1999.

[6] Guang-Huang Gao. Laser radiation injury medical protection. Military Medical Science Press. First edition. 1998.

[7] Feng-Ying Xie. Segmentation and recognition of Dermoscopy for Melanoma images based on computational intelligence. Doctoral Dissertation of Beihang University. 2009.

[8] Menzies SW, Bischof L, Talbot H, et al. The performance of Solar Scan: An automated dermoscopy image analysis instrument for the diagnosis of primary melanoma.Arch Dermatol. 2005; 141: 1388-1396. PMid:16301386 http://dx.doi.org/10.1001/archderm.141.11.1388

[9] Ru-Song Meng, Xiao Meng, Guang Zhao, Rui-Kang Cai, Zhi-Guo Jiang. Diagnostic value of image technology of polari-light dermoscopy in head and face tumors.Chin J Med Aesth\& Cosmet. 2008; 3: 177-179.

[10] Feng-Ying Xie, Shi-Yin Qin, Zhi-Guo Jiang, Ru-Song Mengc. PDE-based Unsupervised Repair of Hair-occluded Information in Dermoscopy Images of Melanoma. Computerized Medical Imaging and Graphics. 2009; 33: 275-282. PMid:19261439 http://dx.doi.org/10.1016/j.compmedimag.2009.01.003

[11] Thomas P. Habif. Clinical dermatology. Peking University Medical Science Edition agency. Fourth edition. 2008; 806. 